\title{
Diversidade e Potencial Micotoxigênico de Aspergillus Spp. no Solo de Sabará (Mg)
}

Vanessa Maria Pereira (I), Fabiana Reinis Passamani (I), Nathasha de Azevedo Lira (I), Sara Maria Chalfoun (II), Fatima Maria de Souza Moreira (I), Luis Roberto Batista (I)

(I) UFLA - Universidade Federal de Lavras (Caixa Postal 3037, Lavras, MG), (II) EPAMIG Empresa de Pesquisa em Agropecuária de Minas Gerais (Caixa postal 176, Lavras, MG)

\section{Resumo}

O solo é considerado um mosaico de micro-habitats devido a sua complexidade e, dessa forma é um dos principais ecossistemas para o desenvolvimento de populações microbianas. Juntamente com a água, o solo é um dos mais importantes contaminantes de alimentos, pois partículas com microrganismos podem ser transportadas por animais, aderir às plantas ou serem disseminadas pela água e pelo ar. O isolamento de Aspergillus spp. do solo é frequente, uma vez que as condições são favoráveis para o seu crescimento. Nesse contexto, o objetivo do trabalho foi investigar a diversidade do gênero Aspergillus no solo, na cidade de Sabará (MG) e avaliar seu potencial micotoxigênico. As amostras de solo $(20 \mathrm{~cm}$ de profundidade) foram coletadas assepticamente e levadas ao laboratório, destas foram realizadas diluições seriadas (10-1 a 10-5) utilizados os meios DG18 e DRBC em triplicata. Após o desenvolvimento das colônias, aquelas que mostraram macromorfologia similar a Aspergillus foram isoladas em MA 2\% e, após o crescimento, foi realizada a identificação morfológica das espécies nos meios MEA e CYA. A temperatura de incubação dos isolados foi de $25^{\circ} \mathrm{C}$, e uma placa a mais por isolado de CYA foi incubada a $37^{\circ} \mathrm{C}$ em BOD. Após 7 dias foram realizadas as medições de colônias e a observação de características microscópicas, conforme o manual de identificação. Estes dados foram usados na chave de identificação. A detecção da produção de micotoxinas foi realizada pelo

\footnotetext{
Referência:

Vanessa Maria Pereira, Fabiana Reinis Passamani, Nathasha de Azevedo Lira, Sara Maria Chalfoun, Fatima Maria de Souza Moreira, Luis Roberto Batista.Diversidade e Potencial Micotoxigênico de Aspergillus Spp. no Solo de Sabará (Mg). In: Anais do $12^{\circ}$ Congresso Latinoamericano de Microbiologia e Higiene de Alimentos - MICROAL 2014 [= Blucher Food Science Proceedings, num.1, vol.1]. São Paulo: Editora Blucher, 2014. 
método Plug Agar em placas de cromatografia de camada delgada, onde as micotoxinas analisadas foram Ocratoxina A e Aflatoxinas B1, B2, G1 e G2. Como resultado, foram obtidos 41 isolados, sendo estes: $21 \mathrm{~A}$. parasiticus; 9 A. niger; 4 A. carbonarius; 4 A. flavus; 1 A. sulphureus; 1 A. ochraceoroseus e 1 sem identificação até o momento. Quanto à produção de micotoxinas, todos isolados de A. parasiticus produziram Afla B1, B2, G1 e G2; todos isolados de A. flavus produziram Afla B1 e B2 e todos isolados de A. carbonarius produziram Ocratoxina $\mathrm{A}$, os demais isolados não produziram nenhuma das micotoxinas analisadas. O número de isolados de Aspergillus encontrados no solo é relativamente baixo quando comparado a amostras de alimentos, como grãos por exemplo. Isso provavelmente é devido à competição, que no solo é mais expressiva. A produção das micotoxinas corresponde ao encontrado em outros trabalhos na literatura.

Palavras-Chave: Aspergillus, Ocratoxina A, Aflatoxina, Solo, Identificação Agência de Fomento: FAPEMIG 